\title{
Whole exome sequencing confirms the clinical diagnosis of Marfan syndrome combined with X-linked hypophosphatemia
}

\author{
Xunlun Sheng ${ }^{1 *}{ }^{*}, X^{\prime}$ ue Chen ${ }^{2 \dagger}$, Bo Lei $^{3 \dagger}$, Rui Chen ${ }^{4}$, Hui Wang ${ }^{4}$, Fangxia Zhang ${ }^{1}$, Weining Rong ${ }^{1}$, Ruoshui Ha ${ }^{5}$, \\ Yani Liu', Feng Zhao ${ }^{6}$, Peizeng Yang ${ }^{3}$ and Chen Zhao ${ }^{2 *}$
}

\begin{abstract}
Background: To determine the genetic lesions and to modify the clinical diagnosis for a Chinese family with significant intrafamilial phenotypic diversities and unusual presentations.

Methods: Three affected patients and the asymptomatic father were included and received comprehensive systemic examinations. Whole exome sequencing (WES) was performed for mutation detection. Structural modeling test was applied to analyze the potential structural changes caused by the missense substitution.

Results: The proband showed a wide spectrum of systemic anomalies, including bilateral ectopia lentis, atrial septal defect, ventricular septal defect, widening of tibial metaphysis with medial bowing, and dolichostenomelia in digits, while her mother and elder brother only demonstrated similar skeletal changes. A recurrent mutation, PHEX p.R291*, was found in all patients, while a de novo mutation, FBN1 p.C792F, was only detected in the proband. The FBN1 substitution was also predicted to cause significant conformational change in fibrillin-1 protein, thus changing its physical and biological properties.

Conclusions: Taken together, we finalized the diagnosis for this family as X-linked hypophosphatemia (XLH), and diagnosed this girl as Marfan syndrome combined with XLH, and congenital heart disease. Our study also emphasizes the importance of WES in assisting the clinical diagnosis for complicated cases when the original diagnoses are challenged.
\end{abstract}

\section{Background}

Marfan syndrome (MFS; MIM 154700), characterized by complicated manifestations in multiple organ systems with high degrees of clinical diversity, is one of the most common autosomal dominant inherited connective tissue diseases with a prevalence of 1 in 5,000 [1]. Cardinal MFS features involve the ocular, skeletal, and cardiovascular systems, of which ectopia lentis and

\footnotetext{
*Correspondence: shengxunlun@163.com; dr_zhaochen@163.com

${ }^{\dagger}$ Xunlun Sheng, Xue Chen and Bo Lei are contributed equally to this work.

${ }^{1}$ Department of Ophthalmology, Ningxia Eye Hospital, Ningxia People's

Hospital, Ningxia, China

${ }^{2}$ State Key Laboratory of Reproductive Medicine, Department

of Ophthalmology, The First Affiliated Hospital of Nanjing Medical

University, Nanjing, China

Full list of author information is available at the end of the article
}

aortic aneurysm are given special clinical significance [2]. Excessive liner growth of the long bones and joint laxity are hallmarks for the skeletal systems. By far, mutations in two genes have been implicated in the etiology of MFS, including the fibrillin 1 (FBN1; MIM 134797) gene and the transforming growth factor beta receptor II (TGFBR2; MIM 190182) gene. Most MFS cases are caused by FBN1 mutations [3], whereas the TGFBR2 mutation has only been found correlated with MFS in a French family [4]. The diagnosis of MFS is usually based on both clinical signs and genetic findings [2].

Familial hypophosphatemic rickets (FHR) can be transmitted via all three types of Mendelian inheritance pattern. Autosomal dominant hypophosphatemic rickets (ADHR; MIM 193100) usually correlates with mutations in the fibroblast growth factor 23 (FGF23; MIM 605380) 
gene [5], whereas mutations in two other genes are implicated in causing the autosomal recessive form, including the dentin matrix acidic phosphoprotein 1 (DMP1; MIM 600980) gene associated with autosomal recessive hypophosphatemic rickets-1 (ARHR1; MIM 241520) [6], and the ectonucleotide pyrophosphatase/phosphodiesterase 1 (ENPP1; MIM 173335) gene related to autosomal recessive hypophosphatemic rickets-2 (ARHR2; MIM 613312) [7]. X-linked hypophosphatemia (XLH; MIM 307800), presenting a prevalence of 1 in 20,000, is the most common form of familial hypophosphatemic rickets (FHR), which is a dominant disorder biochemically featured by hypophosphatemia caused by renal phosphate wasting with normal or low 1,25-dihydroxyvitamin D concentrations [8]. Low serum phosphate concentration and reduced tubular resorption of phosphate corrected for glomerular filtration rate (TmP/GFR) are characteristic for XLH [9]. Comprehensive diagnoses for XLH need are based on clinical findings, radiographic signs, laboratory tests and genetic analyses.

The recently developed next-generation sequencing (NGS) approach enables parallel sequencing of a large panel of candidate genes with high-efficiency, and has therefore been proved as an efficient tool for the molecular diagnosis of multiple diseases [10]. NGS includes whole exome sequencing (WES), targeted gene capture array sequencing and whole sequencing for mapped choromsomal region. Herein, we report the application of WES to detect the disease causative mutation for a Chinese family showing complicated clinical manifestations with significant intrafamilial diversity.

\section{Methods}

\section{Study subjects and clinical assessments}

Four participants from family XLHO1 of Hui ethnicity, including three patients and an unaffected family member, were recruited from the People's Hospital of Ningxia Hui Autonomous Region (Figures 1a, 2a). Our study conformed to the Declaration of Helsinki, and was approved and prospectively reviewed by local ethics committee. Written informed contents were obtained from all participants or their legal guardians before their enrollments. Peripheral blood samples were collected from all four participants using $5 \mathrm{~mL}$ tubes with ethylene diamine tetraacetic acid (EDTA), and a QIAmp DNA blood kit (Qiagen, Valencia, CA) was used for genomic DNA extraction. Family history was carefully reviewed. Medical and personal history was obtained from each participant. Detailed systemic evaluations and laboratory tests were performed on all included family members, including ophthalmic, radiological, cardiological examinations. Additionally, another 100 healthy controls free of MFS and XLH from the same ethnic group were also recruited with their blood samples collected [11-14].

\section{Exome sequencing and bioinformatics analysis}

Exome sequencing was employed on all three patients of this family, including patients I:2, II:1 and II:2, to identify the disease causative mutation for this family. One $\mu \mathrm{g}$ genomic DNA sample for each patient was sheared into 300-500 bp fragments, ligated with Illumina Y shaped adaptors, purified using the Agencourt AMPure SPRI beads, and amplified through ligation-mediated

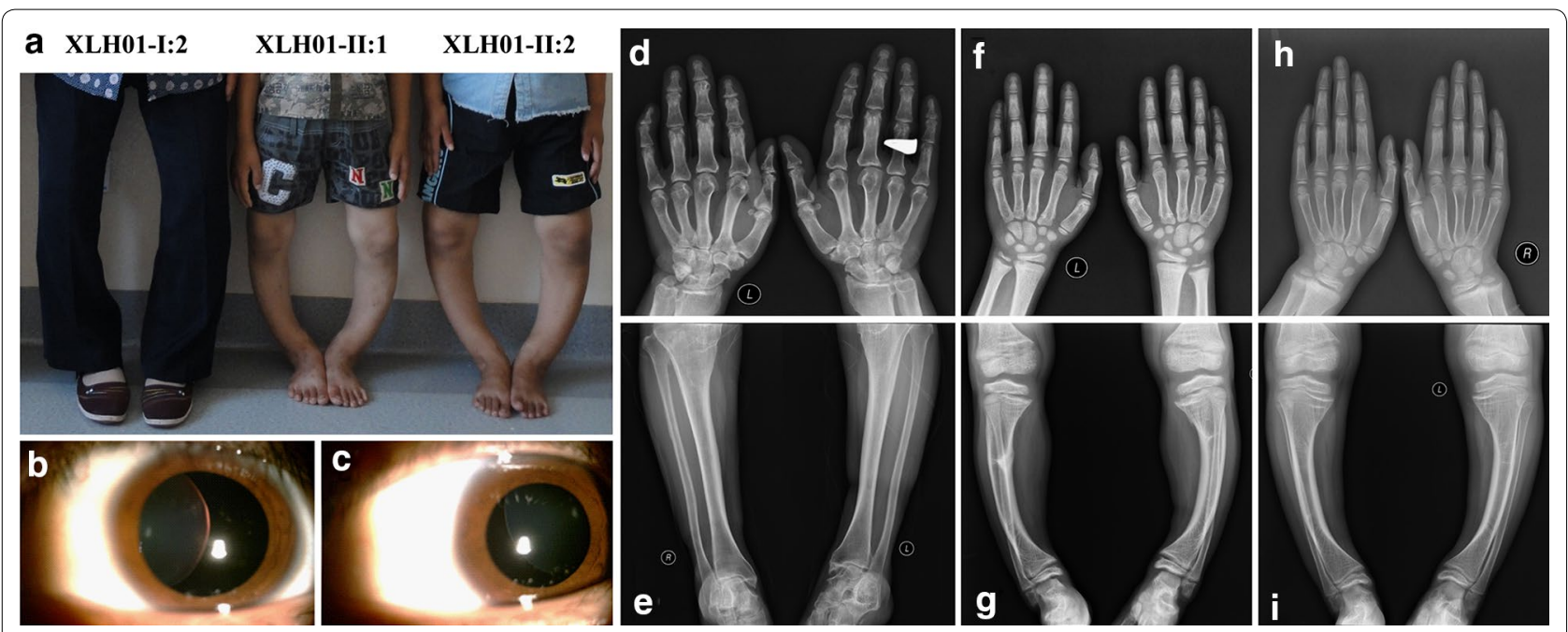

Figure 1 Clinical presentation for family XLH01. a Medial bowing in the patients XLH01-I:2, XLH01-II:1 and XLH01-II:2. b, c Anterior segment photography indicates dislocation of lens toward nasal superior side in both eyes. $\mathbf{d}$-i Radiographic findings reveal dolichostenomelia in the digits of patient XLH01-II:2 (h), but not in patient XLH01-l:2 (d) or XLH01-\|:1 (f). Widening of both proximal and distal tibial metaphysis in patients XLH01-Il:1 $(\mathbf{f}, \mathbf{g})$ and XLH01-II:2 (h, i) are shown. Medial bowing is found in all three patients $(\mathbf{e}, \mathbf{g}, \mathbf{i})$. 


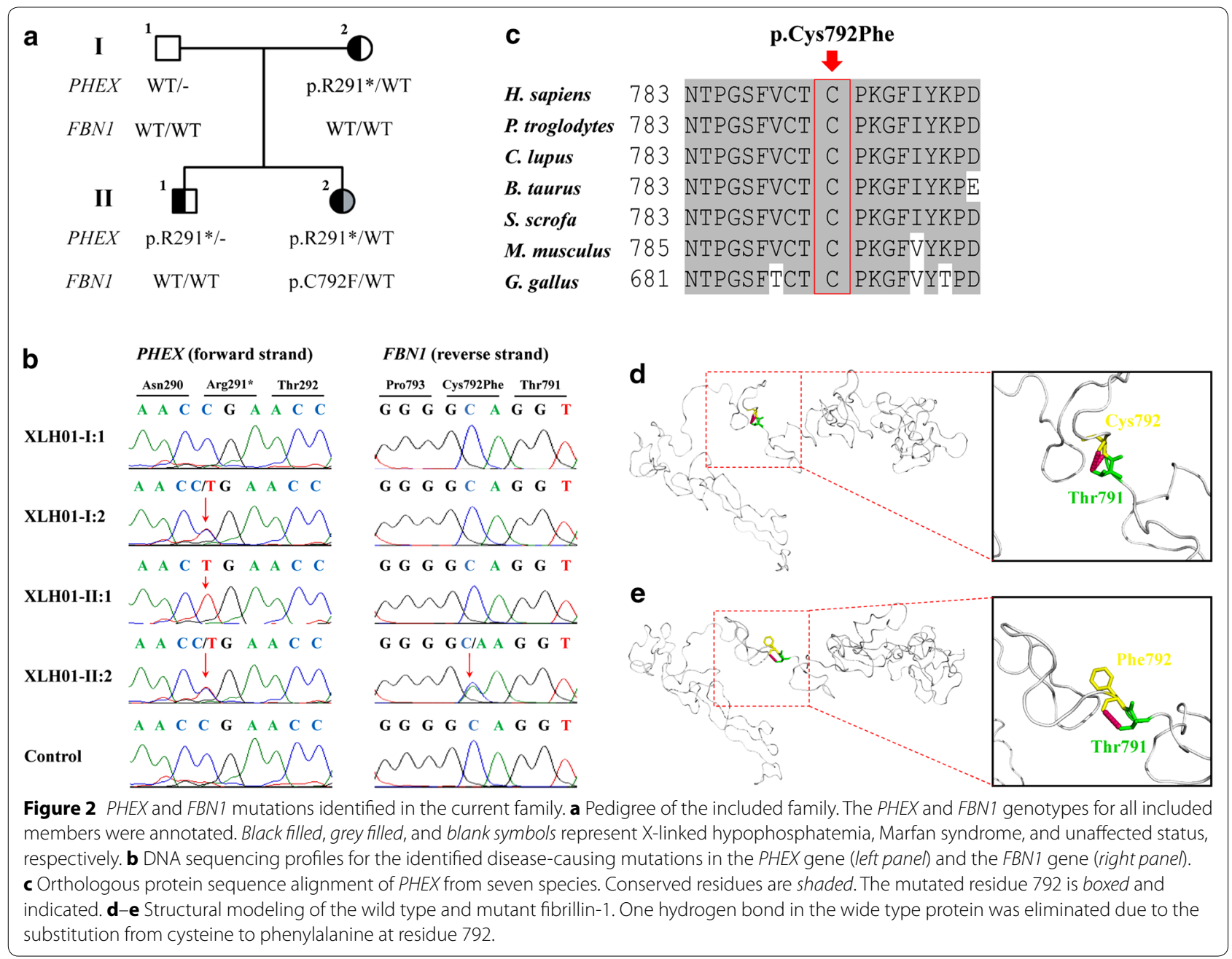

polymerase chain reaction. The SeqCap EZ Hybridization and Wash kit (Roche NimbleGen, Madison, WI) covering 44.1 megabases $(\mathrm{Mb})$ was then employed for enrichment of over 20,000 genes per the manufacture's protocols. The post-capture libraries were quantified using Pico green assay and then sequenced on an Illumina Hiseq 2000 machine as indicated previously [15].

Data were then processed as described previously [15]. Annotations of genes and exons for SeqCap EZ Human Exome Library v2.0 were conducted based on the national center of biotechnology information (NCBI) RefSeq project (Jan 2010), the Consensus CDS (CCDS; Sept 2009) and miRBase (v.14, Sept 2009). Sequence reads were aligned to the NCBI human reference genome (NCBI build 37.1) for SNP analysis with SOAP (http:// www.soap.genomics.org.cn), and for Indel detection using BWA (http://www.bio-bwa.sourceforge.net/). Coverage and depth calculations were further performed as detailed previously [15]. Detected variants were then filtered against the following databases, including 1000 Genome Project (ftp://ftp.1000genomes.ebi.ac.uk/vol1/ ftp), dbSNP135 (http://www.hgdownload.cse.ucsc.edu/ goldenPath/hg19/database/snp135.txt.gz.), Exome Variant Server (http://www.evs.gs.washington.edu/EVS/), NIEHS Exome Sequencing database (http://www.evs. gs.washington.edu/niehsExome/), ExAC Browser (http:// www.exac.broadinstitute.org/), and an internal control database including 997 exomes [16]. We used a cut-off of minor allele frequency (MAF) $0.1 \%$ to reveal variations shared by all three patients and de novo variants carried by the proband girl. An additional cut-off of $1 \%$ was also set to seek for homozygous and compound heterozygous variants that may help to explain the sole phenotypes of the proband girl.

\section{Sanger sequencing and in silico analysis}

Sanger sequencing was then conducted for mutation verification and prevalence test in 100 unrelated 
controls using a previously defined protocol [17]. Information of primers was presented in Additional file 1: Table S1. Evolutionary conservation of the mutated amino acids was assessed via Vector NTI Advance 11 (Invitrogen, Grand Island, NY) through alignment of the fibrillin-1 orthologous protein sequences of the following species: Homo sapiens (ENSP00000368682), Pan troglodytes (ENSPTRP00000037269), Canis lupus familiaris (ENSCAFP00000019648), Bos taurus (ENSBTAP00000000540), Sus scrofa (ENSSSCP00000030440), Mus musculus (ENSMUSP00000078863), and Gallus gallus (ENSGALP00000026360). SIFT Human Protein DB (http://www.sift.jcvi.org/) and PolyPhen-2 (http://www. genetics.bwh.harvard.edu/pph2/) online softwares were used to detect the potential pathogenic impacts of the mutation. Crystal structures of the wide type and mutant phosphate-regulating neutral endopeptidase were obtained with SWISS-MODEL online server (http:// www.swissmodel.expasy.org/). Predicted structures were displayed with PyMol software (version 1.5).

\section{Results}

\section{Clinical assessments}

In June, 2011, the 8-year-old proband (XLH01-II:1) was first referred to our ophthalmic clinic for blurred vision over the past 2 years. She was born at term to a 34-yearold woman after an uneventful full-term pregnancy and delivery. Her best corrected visual acuities (BCVAs) reached 0.1 for the right eye and 0.25 for the left eye. Slit-lamp test revealed dislocation of lens toward nasal superior side and hippus in both eyes (Figure 1b, c). Medical records included an atrioseptopexy at age five for her atrial septal defect (ASD). Other than the repaired ASD presentation, ultrasonic cardiogram (UCG) indicated leftto-right shunt flow through a ventricular septal defect (VSD) with the diameter of $4.50 \mathrm{~mm}$. Slight mitral, tricuspid, and pulmonary regurgitations were also detected. Her calculated Z-score for aortic root was -0.54 .

Physical examination on our patient also showed complex skeletal problems. Her height was $117.0 \mathrm{~cm}[<-2$ standard deviation (SD)] and her weight was $30.0 \mathrm{~kg}$ $(>+1$ SD) on admission. Radiographic examinations showed widening of both proximal and distal tibial metaphysis with medial bowing, but dolichostenomelia in her digits were also revealed (Figure 1h). Laboratory analyses indicated hypophosphatemia, elevated serum alkaline phosphatase (ALP) level, and reduced tubular resorption of phosphate corrected for glomerular filtration rate (TmP/GFR) in this patient (Table 1). Serum calcium, intact-parathyroid hormone (iPTH), 25-hydroxyvitamin D3, and 1, 25-dihydroxyvitamin D3 were within the normal range. Her 41-year-old mother (XLH01-I:2) and 10-year-old brother (XLH01-II:2) had no remarkable ophthalmic or cardiac conditions, but showed similar skeletal abnormalities as demonstrated by physical and laboratorial tests with exception of dolichostenomelia in their digits (Figure 1d, g; Table 1). Her brother's height was $113.0 \mathrm{~cm}(<-3 \mathrm{SD})$. Her mother was $145 \mathrm{~cm}$ in height and had osteomalacia, severe ostealgia, and difficulties in walking, but no remarkable change in serum ALP level.

Table 1 Laboratory analyses for family XLHO1 at their initial visit

\begin{tabular}{|c|c|c|c|c|}
\hline Indexes & Normal Range & XLH01-I:2 & XLH01-II:1 & XLH01-II:2 \\
\hline Age (years) & - & 41 & 8 & 10 \\
\hline Sex & - & Female & Male & Male \\
\hline TmP/GFR (mg/dL) & $2.2-3.6^{\oplus} ; 2.9-6.5^{\dagger}$ & 0.43 & 0.85 & 0.92 \\
\hline Serum ALP (U/L) & $40-110$ & 69 & 337 & 589 \\
\hline Serum iPTH $(\mathrm{pg} / \mathrm{mL})$ & $14-72$ & 65.3 & 43.8 & 45.4 \\
\hline Serum 25-hydroxyvitamin D3 (nmol/L) & $5-200$ & 65 & 105 & 95 \\
\hline Serum 1, 25-dihydroxyvitamin D3 (pmol/L) & $60-108$ & 75 & 85 & 88 \\
\hline Serum calcium (mmol/L) & $2.1-2.6^{\bullet} ; 2.25-2.8^{+}$ & 2.36 & 2.38 & 2.38 \\
\hline Serum potassium (mmol/L) & $3.5-5.5$ & 0.79 & 0.91 & 0.92 \\
\hline Serum sodium (mmol/L) & $135-145$ & 140 & 140 & 144 \\
\hline Serum chloride (mmol/L) & $96-106$ & 102 & 102 & 97 \\
\hline Serum phosphonium (mmol/L) & $1.0-1.6^{\oplus} ; 1.3-1.9^{\dagger}$ & 0.79 & 0.91 & 0.92 \\
\hline Serum magnesium (mmol/L) & $0.7-1.1$ & 1.10 & 0.84 & 0.72 \\
\hline Urine amino-acids* & $(-)$ & $(-)$ & $(-)$ & $(-)$ \\
\hline Urine glucose ${ }^{*}$ & $(-)$ & $(-)$ & $(-)$ & $(-)$ \\
\hline Urine protein* & $(-)$ & $(-)$ & $(-)$ & $(-)$ \\
\hline
\end{tabular}

ALP Alkaline phosphatase, iPTH intact-parathyroid hormone and TmP/GFR tubular resorption of phosphate corrected for glomerular filtration rate.

${ }^{*}$ Qualitative test; ${ }^{\circledR}$ for adult; ${ }^{\dagger}$ for child; (-) negative. 
Table 2 Overview of data production

\begin{tabular}{|c|c|c|c|}
\hline Items & XLH01-I:2 & XLH01-II:1 & XLH01-II:2 \\
\hline Raw reads & $52,834,894$ & $65,462,716$ & $60,937,646$ \\
\hline Reads mapped to genome & $50,880,007$ & $63,035,145$ & $58,693,151$ \\
\hline Mapping rate $(\%)$ & 96.30 & 96.29 & 96.31 \\
\hline Initial number of generated SNVs & 178,276 & 207,524 & 187,354 \\
\hline Initial number of generated Indels & 7,632 & 8,235 & 7,815 \\
\hline Mean depth of target region $(X)$ & 28.23 & 37.63 & 35.12 \\
\hline Coverage of target region (\%) & 96.76 & 96.71 & 96.69 \\
\hline Rate of nucleotide mismatch (\%) & 0.29 & 0.26 & 0.23 \\
\hline $\begin{array}{l}\text { Fraction of target covered } \geq 4 \\
\quad X(\%)\end{array}$ & 93.85 & 94.83 & 94.61 \\
\hline $\begin{array}{l}\text { Fraction of target covered } \geq 10 \\
\quad X(\%)\end{array}$ & 75.49 & 80.56 & 79.45 \\
\hline $\begin{array}{l}\text { Fraction of target covered } \geq 20 \\
\quad X(\%)\end{array}$ & 57.48 & 66.88 & 64.97 \\
\hline Duplication rate (\%) & 7.92 & 9.59 & 8.80 \\
\hline Gender test result & $\mathrm{F}$ & $M$ & $\mathrm{~F}$ \\
\hline
\end{tabular}

SNV Single nucleotide variants and Indel insertions and deletions.

The proband was thereafter treated with a surgical removal of the dislocated lens with implantation of an artificial intraocular lens and received amblyopia training after surgery. At the 3-year follow-up, her BCVAs improved to 0.4/0.5 (OD/OS). She also had follow-up visits in the cardiology and pediatric departments and was supplemented with elemental phosphorus and calcitriol. Her ALP level was back to normal at 2-year follow-up with no complication.

\section{Genetic findings}

To assist the clinical diagnosis in this unusual family, we performed whole exome sequencing (WES) on patients XLH01-I:2, XLH01-II:1 and XLH01-II:2 (Table 2 ). The average mean depth of the three tested samples was $33.7 \mathrm{X}$. A total of 24 variants passed the initial filtration process and were confirmed carried by all three patients and absent in the unaffected father (Additional file 1: Table S1). Conversely, a different set of 24 variants with MAF $<0.01 \%$ in the Chinese population present in the proband but not in the brother or mother were selected to confirm paternity of the father. All 24 variants were observed as heterozygotes in the father (Additional file 1: Table S2), a finding that is not likely to occur in the setting of non-paternity. Among the initial 24 post-filtration variants, a recurrent heterozygous/hemizygous mutation in the PHEX gene (c.871C>T [NM_000444]; p.R291* [NP_000435]) was identified in all three affected individuals (Figure 2a, b). This mutation was previously reported in a patient with $\mathrm{XLH}$, and was predicted to generate a premature termination codon at residue 291, which would suspend translation [18]. We therefore believed that this PHEX variation was more likely the disease causing mutation for this family than other identified variants. In addition, a de novo heterozygous mutation in the FBN1 gene (c.2375G>T [NM_000138]) was only called in the affected girl (Figure 2a, b). This mutation would cause the amino acid change from the hydrophilic cysteine to the hydrophobic phenylalanine at residue 792 (p.C792F [NP_000129]). Residue Cys792 was predicted to be highly conserved among multiple species (Figure 1c), and both SIFT (score: 0.02; damaging) and PolyPhen-2 (score: 0.997; probably damaging) softwares suggested the deleterious impact of this substitution. Crystal structural modeling of the wide type and mutant fibrillin-1 (residues 584-950) was constructed on the basis of low-density lipoprotein receptor (protein data bank [PDB] ID: 1N7D) with the sequence similarity of 0.38 (Figure 2d, e) [19]. One hydrogen bond between residue 792 and Thr791 was eliminated due to the substitution from cysteine to phenylalanine, which significantly altered its tertiary structure and would further change relevant protein properties.

\section{Discussion}

MFS and XLH are both systemic diseases but with opposite skeletal changes. MFS and XLH have not been simultaneously reported in any individual before. In our case, the affected girl had a broad spectrum of systemic anomalies, including bilateral ectopia lentis, ASD, VSD, widening of tibial metaphysis with medial bowing, dolichostenomelia in digits, hypophosphatemia, elevated serum ALP level, and reduced TmP/GFR. Similar skeletal and laboratory changes, but except for dolichostenomelia in digits, ocular or cardiac conditions, were also demonstrated by her mother and older brother. The phenotypic complexity and intrafamilial diversity have challenged the original diagnosis for this family, especially for the proband. With the power of WES, we have identified a nonsense mutation PHEX p.R291* shared by all three patients, plus a de novo FBN1 p.C792F exclusively in the proband. The radiological findings, laboratory results, and presence of the nonsense PHEX mutation in all 3 patients suggest the diagnosis of XLH for this family [9]. The bilateral ectopia lentis, dolichostenomelia in digits, and the cysteine substitution in FBN1, the most common type of $F B N 1$ mutation that correlates with moderate phenotypes of presumed MFS, revealed in the proband confer an additional diagnosis of MFS to this patient [2]. Another diagnosis of congenital heart disease (CHD) was also finalized to this proband based on the ASD and VSD presentations. Therefore, the clinical presentations and genetic findings confirm the diagnosis of XLH in this family, and MFS combined with XLH and CHD in the 
8-year-old proband. To the best of our knowledge, this is the first report of the comprehensive diagnosis of MFS, $\mathrm{XLH}$ and CHD in a single patient. Our report also supports the significant role of clinical genetics in the diagnosis of complicated diseases.

Traditional approaches for mutation detection have their limitations, usually resulting into their low diagnostic rate. When compared with traditional methods, NGS has revolutionized the speed and cost for generating large quantities of sequence data [10]. In the present study, mutations were identified via a series of genetic analyses including initial WES, optimized bioinformatics analysis, cosegregation analyses, and mutation screening in control groups [12, 15]. In silico analyses, including the crystal structure modeling tests, also support pathogenicity of identified mutations [13]. With these efforts, our results strongly support that those variants detected are disease-causing mutations rather than rare polymorphisms, and suggests NGS as a promising technology for identifying the novel disease-causing genes associated with monogenic diseases. However, our study also has limitations. WES of the proband alone followed by Sanger sequencing of the variants identified would be a more economic and sufficient strategy for this case.

\section{Conclusion}

In summary, our study not only reports an extremely rare case, but also emphasizes the importance of recently developed approach of WES in the assistance of clinical diagnosis for complicated cases. Genetic counselling and molecular diagnosis would significantly assist clinical diagnoses for such patients with complicated phenotypes, and direct the clinical management.

\section{Additional file}

Additional file 1: Supplementary Tables.

\begin{abstract}
Authors' contributions
$X S, X C$, and $B L$ contributed equally to this report. All authors were involved in managing the patients. $X S, X C$, and $C Z$ wrote the report. $B L, H W$, and $R C$ did the genetic analysis and whole exome sequencing, and $C Z$ reviewed the genetic results. Written consent to publish was obtained. All authors read and approved the final manuscript.
\end{abstract}

\footnotetext{
Author details

${ }^{1}$ Department of Ophthalmology, Ningxia Eye Hospital, Ningxia People's Hospital, Ningxia, China. ${ }^{2}$ State Key Laboratory of Reproductive Medicine, Department of Ophthalmology, The First Affiliated Hospital of Nanjing Medical University, Nanjing, China. ${ }^{3}$ Chongqing Key Laboratory of Ophthalmology, Department of Ophthalmology, Chongqing Eye Institute, The First Affiliated Hospital of Chongqing Medical University, Chongqing, China. ${ }^{4}$ Human Genome Sequencing Center, Baylor College of Medicine, Houston, TX, USA. ${ }^{5}$ Department of Radiology, Ningxia Eye Hospital, Ningxia People's Hospital, Ningxia, China. ${ }^{6}$ Department of Cardiology and Surgery, Tianjin Chest Hospital, Tianjin Medical University, Tianjin, China.
}

\section{Acknowledgements}

The authors thank all patients and their family members for their participation. This work was supported by National Key Basic Research Program of China (973 Program, 2013CB967500); National Natural Science Foundation of China (81222009, 81170856,81160124 and 81260154); Thousand Youth Talents Program of China (to C. Z.); Jiangsu Outstanding Young Investigator Program (BK2012046); Jiangsu Province's Key Provincial Talents Program (RC201149); the Fundamental Research Funds of the State Key Laboratory of Ophthalmology (to C. Z.); Jiangsu Province's Scientific Research Innovation Program for Postgraduates (CXZZ13_0590); and A Project Funded by the Priority Academic Program Development of Jiangsu Higher Education Institutions (PAPD). The funders had no role in study design, data collection and analysis, decision to publish, or preparation of the manuscript.

\section{Compliance with ethical guidelines}

\section{Competing interests}

The authors declare that they have no competing interests.

Received: 7 February 2015 Accepted: 13 May 2015

Published online: 04 June 2015

\section{References}

1. von Kodolitsch Y, Robinson PN (2007) Marfan syndrome: an update of genetics, medical and surgical management. Heart 93:755-760

2. Dietz HC (1993) Marfan Syndrome. GeneReviews. University of Washington, Seattle. http://www.ncbi.nlm.nih.gov/books/NBK1335/

3. Zhao F, Pan X, Zhao K, Zhao C (2013) Two novel mutations of fibrillin-1 gene correlate with different phenotypes of Marfan syndrome in Chinese families. Mol Vis 19:751-758

4. Mizuguchi T, Collod-Beroud G, Akiyama T, Abifadel M, Harada N, Morisaki Tet al (2004) Heterozygous TGFBR2 mutations in Marfan syndrome. Nat Genet 36:855-860

5. White KE, Evans WE, O'Riordan JL, Speer MC, Econs MJ, Lorenz-Depiereux $B$ et al (2000) Autosomal dominant hypophosphataemic rickets is associated with mutations in FGF23. Nat Genet 26:345-348

6. Lorenz-Depiereux B, Bastepe M, Benet-Pages A, Amyere M, Wagenstaller J, Muller-Barth $U$ et al (2006) DMP1 mutations in autosomal recessive hypophosphatemia implicate a bone matrix protein in the regulation of phosphate homeostasis. Nat Genet 38:1248-1250

7. Levy-Litan V, Hershkovitz E, Avizov L, Leventhal N, Bercovich D, ChalifaCaspi $V$ et al (2010) Autosomal-recessive hypophosphatemic rickets is associated with an inactivation mutation in the ENPP1 gene. Am J Hum Genet 86:273-278

8. Cheon CK, Lee HS, Kim SY, Kwak MJ, Kim GH, Yoo HW (2014) A novel de novo mutation within PHEX gene in a young girl with hypophosphatemic rickets and review of literature. Ann Pediatr Endocrinol Metab 19:36-41

9. Ruppe MD (1993) X-Linked Hypophosphatemia. GeneReviews. University of Washington, Seattle. http://www.ncbi.nlm.nih.gov/books/NBK83985/

10. Shendure J, Ji H (2008) Next-generation DNA sequencing. Nat Biotechnol 26:1135-1145

11. Rong W, Chen X, Zhao K, Liu Y, Liu X, Ha S et al (2014) Novel and recurrent MYO7A mutations in Usher syndrome type 1 and type 2. PLoS One 9:e97808

12. Chen X, Liu Y, Sheng X, Tam PO, Zhao K, Rong W et al (2014) PRPF4 mutations cause autosomal dominant retinitis pigmentosa. Hum Mol Genet 23:2926-2939

13. Chen X, Sheng X, Liu X, Li H, Liu Y, Rong W (2014) Targeted next-generation sequencing reveals novel USH2A mutations associated with diverse disease phenotypes: implications for clinical and molecular diagnosis. PLoS One 9:e105439

14. Pan X, Chen X, Liu X, Gao X, Kang X, Xu Q et al (2014) Mutation analysis of pre-mRNA splicing genes in Chinese families with retinitis pigmentosa. Mol Vis 20:770-779

15. Chen X, Zhao K, Sheng X, Li Y, Gao X, Zhang X et al (2013) Targeted sequencing of 179 genes associated with hereditary retinal dystrophies and 10 candidate genes identifies novel and known mutations 
in patients with various retinal diseases. Invest Ophthalmol Vis Sci 54:2186-2197

16. Fu Q, Wang F, Wang H, Xu F, Zaneveld JE, Ren $\mathrm{H}$ et al (2013) Next-generation sequencing-based molecular diagnosis of a Chinese patient cohort with autosomal recessive retinitis pigmentosa. Invest Ophthalmol Vis Sci 54:4158-4166

17. Zhao C, Lu S, Zhou X, Zhang X, Zhao K, Larsson C (2006) A novel locus (RP33) for autosomal dominant retinitis pigmentosa mapping to chromosomal region 2cen-q12.1. Hum Genet 119:617-623
18. Holm IA, Huang X, Kunkel LM (1997) Mutational analysis of the PEX gene in patients with X-linked hypophosphatemic rickets. Am J Hum Genet 60:790-797

19. Rudenko G, Henry L, Henderson K, Ichtchenko K, Brown MS, Goldstein JL et al (2002) Structure of the LDL receptor extracellular domain at endosomal pH. Science 298:2353-2358

\section{Submit your next manuscript to BioMed Central} and take full advantage of:

- Convenient online submission

- Thorough peer review

- No space constraints or color figure charges

- Immediate publication on acceptance

- Inclusion in PubMed, CAS, Scopus and Google Scholar

- Research which is freely available for redistribution

Submit your manuscript at

www.biomedcentral.com/submit

() BioMed Central 